\title{
3D MESO-SCALE MODELLING OF CONCRETE MATERIAL IN SPALL TESTS
}

\author{
${ }^{1}$ Gang Chen, ${ }^{2}$ Yifei Hao ${ }^{*}$ and ${ }^{2}$ Hong Hao \\ ${ }^{1}$ Institute of Systems Engineering, CAEP, Sichuan, China, 621900 \\ ${ }^{2}$ Tianjin University and The University of Western Australia Joint Research Centre of \\ Protective Structures \\ School of Civil and Resource Engineering, The University of Western Australia \\ 35 Stirling Highway, Crawley, WA 6009, Australia
}

\begin{abstract}
Tensile strength is one of the key factors of concrete material that need be accurately defined in analysis of concrete structures subjected to high-speed impact loads. Dynamic tensile strength of concrete material is usually obtained by conducting laboratory tests such as direct tensile test, Brazilian splitting test and spall test. Concrete is a heterogeneous material with different components, but is conventionally assumed to be homogeneous, i.e. cement mortar only, in most previous experimental or numerical studies. The aggregates in concrete material are usually neglected owing to testing limitation and numerical simplification. It has been well acknowledged that neglecting coarse aggregates might not necessarily give accurate concrete dynamic material properties. In the present study, a 3D mesoscale model of concrete specimen with consideration of cement mortar and aggregates is developed to simulate spall tests and investigate the behaviour of concrete material under high strain rate. The commercial software LS-DYNA is used to perform the numerical simulations of spall tests. The mesh size sensitivity is examined by conducting mesh convergence tests. The reliability of the numerical model in simulating the spall tests is verified by comparing the numerical results with the experimental data from the literature. The influence of coarse aggregates on the experimental test results is studied. The wave attenuation in concrete specimen is analysed, and empirical equations are proposed for quick assessment of the test data to determine the true dynamic tensile strength of concrete material. The contributions of aggregates to dynamic strength in spall tests are quantified for modifying the test results based on mortar material in the literature.
\end{abstract}

Keywords: Concrete, dynamic tension, spall test, meso-scale model, wave dispersion, coarse aggregates

\section{INTRODUCTION}

Concrete is a commonly used construction material in both civil and defence structures. In general, concrete exhibits much lower strength in tension as compared to that in compression. Military structures usually need be designed to

\footnotetext{
*Corresponding author. E-mail: hao.yifei@ outlook.com
} 
1 resist blast loads. Moreover, due to the increased terrorist activities and rapid 2 industrial development and urbanization, civilian structures might also experience 3 high-rate loads such as blast or vehicle impacts. The primary failure modes of tensile force, and concrete structure is more vulnerable to tensile failure. Concrete structures such as slabs, walls, beams and columns in practice all experience the combined actions of compression, tension and shear forces. Although concrete tensile strength is usually neglected in static design analysis as it is relatively small as compared to its compressive strength and only reinforcement bars are considered to resist the tensile force, it is an important parameter because it often governs the concrete structure failure. For example, tensile strength controls the crack opening in concrete structures under static loading. When the concrete structure is under dynamic loading, a stress wave is induced in the structure, which induces both tensile and compressive stresses in the structure and tensile strength governs the structural failure, such as the spalling failure of concrete beam and slab under impact loading. Understanding concrete tensile strength is essential for reliable numerical modelling and safe concrete structure designs. The difference of considering different tensile strengths of concrete in simulating reinforced concrete panel response to blast load has been demonstrated by Jones et al. (2009). Based on their study, it was concluded that ignoring strain rate effects overestimated the structural responses. Therefore it is important to understand the behaviour of concrete material in tension at high strain rate for more reliable design and analysis of protective structures subjected to impact and blast loadings.

There are several experimental methods such as direct tensile test (Tedesco et al. 1991; Yan and Lin 2006), Brazilian splitting test (Gomez et al. 2001) and spall test (Brara et al. 2001, Schuler et al. 2006) that have been developed and commonly used to obtain the dynamic tensile strength of brittle materials such as mortar, concrete and rock. The direct tensile test and Brazilian splitting test, normally instrumented with a split Hopkinson pressure bar (SHPB) setup, are able to study the behaviour of concrete materials under dynamic tension for strain rates up to the $20 \mathrm{1} / \mathrm{s}$. However, for concrete structural elements experiencing high-speed impact or close-in blast loads, the tensile strain rate can be a magnitude higher, i.e., $10^{2} 1 / \mathrm{s}$, than the achievable strain rate from the direct tensile and Brazilian splitting tests. Besides the limitations in achieving higher strain rates, it is not always easy for concrete specimens to achieve stress uniformity, an essential requirement for reliable presentation of SHPB test results, in direct tensile and Brazilian splitting tests. Therefore other test methods have constantly been explored by different researchers.

The method of spall test is known from plate impact tests and was adapted to Hopkinson bar experiments. An analysis of the spall test as a method to determine the dynamic tensile strength of brittle materials was presented by Gálvez Díaz-Rubio et al. (2002). The main apparatus of the spall experimental technique includes a striker bar, a Hopkinson incident bar and a slender rod specimen as shown in Fig. 1. The incident compressive stress wave, produced by a projectile striking on the incident bar, propagates along the bar. When it approaches the bar-specimen interface, part of it is transmitted into the specimen rod whereas the rest is reflected. When the transmitted compressive stress wave arrives at the free end 
1 of the specimen, it is reflected as a tensile stress which may lead to fracture of 2 the specimen made of materials that have much lower strength in tension than 3 that in compression. When fracture occurs, the fractured layer closed to the free 4 end traps an amount of momentum and flies off, which is described as the spall

phenomenon. Because spall is a wave-induced dynamic fracture, which does not require the stress uniformity, and is able to achieve a higher strain rate up to a magnitude of $10^{2} 1 / \mathrm{s}$, it has been more and more widely used in testing brittle materials in relatively higher strain rate range. However, there is no direct measurement method for the spall strength. Several indirect methods have been proposed to acquire the material spall strength. One of them analyzes the onedimensional wave, with consideration of geometric wave attenuation, to predict the reflected tensile stress wave from the incident compressive wave at the free end of the specimen, and the tensile strength of the concrete is defined as the level of the maximum tensile stress reached at the position of the first fracture. Another method is derived from the plate-impact technique. The spall strength is obtained from the velocity of the rear face of the specimen as $\rho C \Delta V_{p b} / 2$, where $\rho$ is the density of the material, $C$ is the one-dimensional wave velocity, and $\Delta V_{p b}$, called pullback velocity, is the difference of the velocity between the maximum value and the rebound velocity. The strain rate in the test is estimated from the strength, the Young's modulus and the interval of the time instants between the one when tensile stress occurs and the time when the failure takes place.

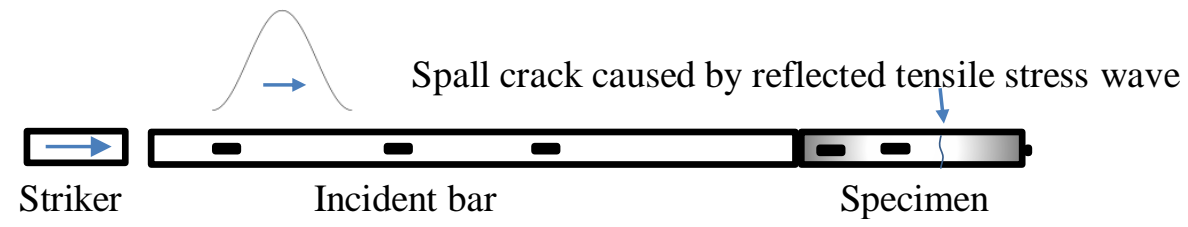

Figure 1: Configuration of spall test

A number of spall tests on cementitious materials have been reported (Daimaruya 1997; Klepaczko and Brara 2001; Brara et al. 2001,2006; Wu et al. 2005; Schuler et al. 2006; Weerheijm and Doormaal 2007; Zhang et al. 2009; Erzar and Forquin 2010). Most of these tests used cement mortar only in preparing the specimen although the test results were used to derive the concrete material properties. Some tests considered concrete-like material (micro-concrete) in which sand, or so-called fine aggregates, is used to prepare the specimen in studies of dynamic concrete material properties. Because different components in a concrete mix have different material properties, these testing data may not necessarily give accurate predictions of concrete material properties. For example, Yan and Lin (2006) conducted direct tensile test and observed that the fracture surfaces of the specimens became more and more flattened with the increasing strain rate; and an increasing number of coarse aggregates were broken along the fracture surface. They concluded that a higher stress level is needed to break aggregates into pieces along the fracture surface. Their test results clearly demonstrated the influences of aggregates on concrete dynamic tensile strength, and using test results of mortar material to represent concrete material property might lead to inaccurate concrete tensile strength prediction. Numerical simulations of splitting tests (Zhou and Hao 2008a) and direct tensile tests (Hao et al 2012) of concrete materials with meso-scale model that includes aggregates and mortar matrix also clearly show the influences of coarse aggregates under 
1 high-speed impact. The latter studies concluded that damage of high-strength 2 aggregates contributes to the tensile strength increment of concrete material. 3 Therefore it is necessary to consider the coarse aggregates in both experimental and numerical studies in order to derive more reliable concrete dynamic tensile strength.

The present study develops three dimensional meso-scale models of concrete specimens with consideration of mortar matrix and coarse aggregates to investigate the behaviour of concrete material in spall tests. The commercial software package LS-DYNA is employed to perform the numerical simulations. The reliability of the numerical model in simulating the spall tests is verified by comparing the numerical results with the published experimental data (Wu et al. 2005). The influence of the heterogeneity on the test results is discussed. Based on numerical simulation results, an 'abnormal' phenomenon observed in the test, that is, rather than attenuation, the reflected tensile wave increase with distance (Wu et al. 2005), is explained. The wave dispersion effect is also analysed, and the wavelength with negligible wave dispersion effect is identified and used in parametric simulations. Based on the numerical results, empirical relations are proposed to quantify the influence of aggregates and to modify the test results from mortar material to derive a more accurate concrete dynamic tensile strength in spall tests.

\section{MATERIAL MODEL}

The plasticity model for concrete in LS-DYNA developed by Malvar et al (1997) is adopted to model the mortar and aggregates in the simulation (LSTC 2007). This model uses three shear failure surfaces with the consideration of damage and strain rate effects. The material model is briefly introduced below.

\subsection{Three-curve failure surface}

The plasticity concrete model, termed as CONCRETE_DAMAGE_REL3 (Mat_072R3) in LS-DYNA, is based on the PSEUDO TENSOR (Mat_16) using three independent strength surfaces, namely, an initial yield surface, a maximum failure surface and a residual surface. They are defined independently with eight material parameters, $a_{i}$, as

$$
\Delta \sigma_{m}=a_{0}+\frac{p}{a_{1}+a_{2} p}
$$

$$
\Delta \sigma_{r}=\frac{p}{a_{1 f}+a_{2 f} p}
$$

$$
\Delta \sigma_{y}=a_{0 y}+\frac{p}{a_{1 y}+a_{2 y} p}
$$

where $\Delta \sigma_{m}, \Delta \sigma_{r}$ and $\Delta \sigma_{y}$ represent the maximum, residual and yield failure surfaces; and $p=-\sigma_{k k} / 3$ is the pressure. 

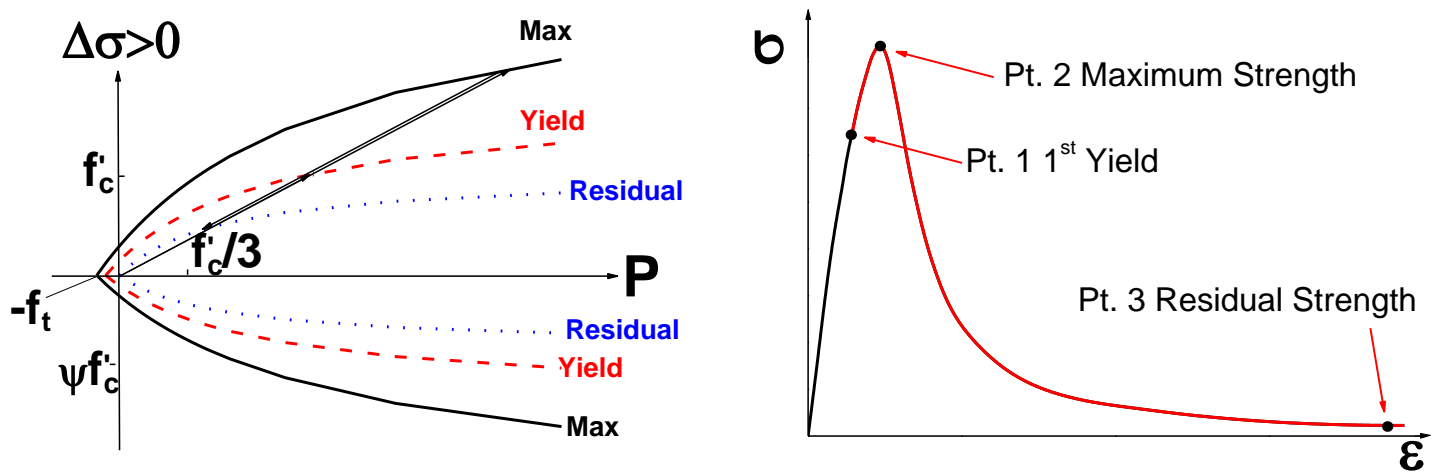

Figure 2: Three failure surfaces (Malvar et al. 1997)

After the stress reaches the initial yield surface but below the maximum failure surface, the current stress is calculated by means of a linear interpolation between the two surfaces, i.e.,

$$
\Delta \sigma=\eta\left(\Delta \sigma_{m}-\Delta \sigma_{y}\right)+\Delta \sigma_{y}
$$

After reaching the maximum failure surface, the current post-failure stress is similarly interpolated between the maximum and the residual stress surfaces, i.e.,

$$
\Delta \sigma=\eta\left(\Delta \sigma_{m}-\Delta \sigma_{r}\right)+\Delta \sigma_{r}
$$

where $\eta$ is a parameter, which indicates the relative amount of damage and the current failure surface region, and is a user-input function of the effective plastic strain damage $\lambda$.

With this model, during initial loading or reloading, the deviatoric stresses remain elastic until the stress point reaches the initial yield surface. The deviatoric stresses can then increase further until the maximum yield surface is reached. Beyond this stage the response can be perfectly plastic or soften to the residual yield surface as shown in Fig. 2.

\subsection{Damage function}

The shear damage function is defined as

$$
\lambda= \begin{cases}\int_{0}^{\bar{\varepsilon}^{p}} \frac{d \bar{\varepsilon}^{p}}{r_{f}\left(1+p / r_{f} f_{t}\right)^{b_{1}}} & \text { when } p \geq 0 \\ \int_{0}^{\bar{\varepsilon}^{p}} \frac{d \bar{\varepsilon}^{p}}{r_{f}\left(1+p / r_{f} f_{t}\right)^{b_{2}}} & \text { when } p<0\end{cases}
$$

20 where $d \bar{\varepsilon}_{p}$ is the effective plastic strain increment given by $d \bar{\varepsilon}_{p}=\sqrt{\frac{2}{3} d \varepsilon_{i j}^{p} d \varepsilon_{i j}^{p}} ; b_{1}$ and $b_{2}$ are damage scaling parameters for the cases of compression and tension, respectively; and $r_{f}$ is the dynamic increase factor that accounts for strain rate effects.

A volumetric plastic strain increment $\Delta \lambda$ is added into the (total) damage function $\lambda$, 
where $b_{3}$ is a user-defined scalar multiplier, $k_{d}$ denotes an internal scalar multiplier, $\varepsilon_{v}$ represents the volumetric strain, and $\varepsilon_{v \text {.yield }}$ is the volumetric strain at yield. The scalar $f_{d}$ is used to restrict the effect of the volumetric damage only to stress conditions close to a triaxial tensile path.

\subsection{Equation of state}

The volumetric behaviour is governed by a compaction curve or an equation of state (EOS), which describes the compressive behaviour of the material, the EOS provides the pressure, $p$, as a function of the volumetric strain and the internal energy. A tabulated compaction model (EOS 8) is used with Mat_072R3, which is linear in the internal energy. The pressure is defined by

$$
p=C\left(\varepsilon_{v}\right)+\gamma T\left(\varepsilon_{v}\right) E
$$

throughout the loading phase. The function $C\left(\varepsilon_{v}\right)$ is provided as a series of $\left(p, \varepsilon_{v}\right)$ pairs in the keyword input file. The EOS prescribes a set of pressures, unloading bulk modules and volumetric strains.

The automatic model parameter generation in LS-DYNA version 971 is used in the simulation. The input material parameters used in the present study are listed in Table 1.

\begin{tabular}{ccc}
\hline Parameters & mortar & aggregate \\
\hline Density $\left(\mathrm{kg} / \mathrm{m}^{3}\right)$ & 2100 & 2600 \\
Poisson's ratio & 0.19 & 0.16 \\
Strength(MPa) & 34 & 160 \\
\hline
\end{tabular}

Table 1: Material parameters of mortar and aggregate

\subsection{Strain rate effect}

The strain rate effect on the material strength is described by the dynamic increase factor (DIF). In the simulation, the compressive DIF relations for mortar matrix are adopted from Hao and Hao (2011), which have the lateral inertia confinement effect removed as verified by the experimental data (Hao et al. 2013). The tensile DIFs used for mortar matrix are adopted from Malvar et al. (1998).

for $\quad \dot{\varepsilon_{d}} \leq 30 s^{-1}$

$$
C D I F=0.8988\left(\log \dot{\varepsilon}_{d}\right)^{2}-2.8255\left(\log \dot{\varepsilon}_{d}\right)+3.4907
$$

for

$30 s^{-1}<\dot{\varepsilon_{d}}<1000 s^{-1}$

$T D I F=\left(\dot{\varepsilon_{d}} / \dot{\varepsilon_{t s}}\right)^{\delta}$

for

$\dot{\varepsilon_{d}} \leq 1 s^{-1}$

$T D I F=\beta\left(\dot{\varepsilon_{d}} / \dot{\varepsilon_{t s}}\right)^{1 / 3}$

for $30 s^{-1}<\dot{\varepsilon_{d}}<1000 s^{-1}$

where $\delta=1 /\left(1+8 f_{c s} / f_{c 0}\right), \log \beta=6 \delta-2, f_{c s}$ is the static compressive strength and $f_{c 0}=10 \mathrm{MPa}, \varepsilon_{t s}^{\cdot}=10^{-6} s^{-1}$. 
The equations of the compressive and the tensile DIFs for the coarse aggregates derived by Hao and Hao (2013) are adopted in this study and given below.

$$
\begin{array}{llcl}
C D I F=0.0187\left(\log \dot{\varepsilon}_{d}\right)+1.2919 & \text { for } & 1 s^{-1} \leq \dot{\varepsilon}_{d} \leq 220 \mathrm{~s}^{-1} \\
C D I F=1.8547\left(\log \dot{\varepsilon}_{d}\right)^{2}-7.9014\left(\log \dot{\varepsilon}_{d}\right)+9.6674 & \text { for } & & 220 \mathrm{~s}^{-1} \leq \dot{\varepsilon}_{d} \leq 1000 \mathrm{~s}^{-1} \\
\text { TDIF }=0.0598\left(\log \dot{\varepsilon}_{d}\right)+1.3588 & \text { for } & & 10^{-6} \mathrm{~s}^{-1} \leq \dot{\varepsilon}_{d} \leq 0.1 \mathrm{~s}^{-1} \\
T D I F=0.5605\left(\log \dot{\varepsilon}_{d}\right)^{2}+1.3871\left(\log \dot{\varepsilon}_{d}\right)+2.1256 & \text { for } & & 0.1 \mathrm{~s}^{-1} \leq \dot{\varepsilon}_{d} \leq 50 \mathrm{~s}^{-1}
\end{array}
$$

Because there are only very limited test data available, the tensile DIF is set to have a constant value when strain rate exceeds $501 / \mathrm{s}$ to avoid overestimation.

\section{3D MESO-SCALE MODEL OF CONCRETE}

\subsection{Generation algorithm of coarse aggregate particles}

Previous studies have proven that models with circular aggregates yield reliable simulations of responses of concrete specimens under static and impact loads (Zhou and Hao 2008b; Hao et al. 2009). To simplify the numerical model, in the present study, coarse aggregates are assumed to have circular shape with random size and distribution in concrete specimen.

The aggregate particle size distribution is assumed to follow Fuller's curve, which defines the grading of aggregate particles for optimum density and strength of the concrete mixture. Fuller's curve, as shown in Fig. 3, can be expressed by the equation

$$
p(d)=100\left(\frac{d}{d_{\max }}\right)^{n}
$$

where $p(d)$ is the cumulative percentage of aggregates passing a sieve with aperture diameter $d, d_{\max }$ is the maximum size of aggregate particle. $n$ is the exponent of the equation, varying from 0.45 to 0.7 , which is taken as 0.5 in the present study.

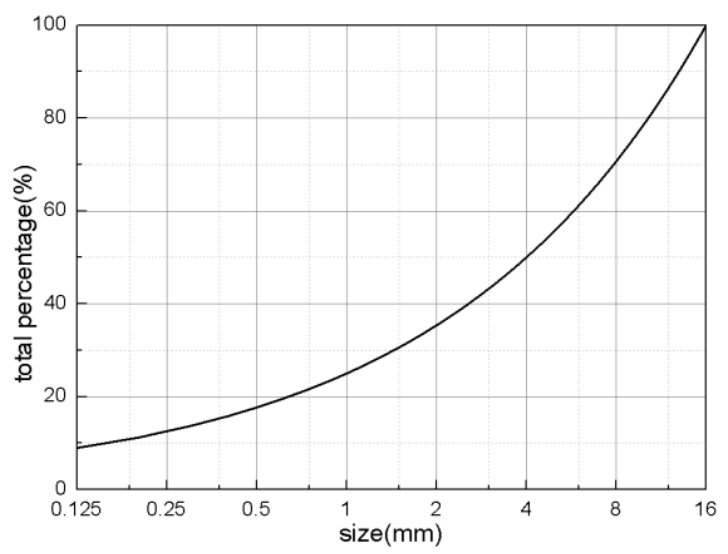

Figure 3: Fuller's grading curve

In this study, a 3D grading concrete meso-scale model is developed. The size of coarse aggregate is calculated according to Fuller's curve defined in Equation (17). 
1 An algorithm of aggregate generation and overlap judgment is proposed to generate coarse aggregate particles. A Fortran-program is developed to generate the random distribution of the aggregates. The programming procedure is summarized in the following steps:

Step 1: Randomly generate the diameter of an aggregate within the size range calculated according to Fuller's curve;

Step 2: Randomly generate the position of the aggregate within the range of the specimen;

Step 3: Check whether the boundary condition is satisfied to avoid overlapping among aggregates;

Step 4: If the generated aggregate satisfies the boundary conditions, record the parameters of the aggregate; otherwise delete the aggregate and perform a new generation until the generated aggregate satisfies the boundary conditions and is properly placed;

Step 5: Repeat the above steps until a certain percentage of aggregates is reached.

\subsection{Mapping algorithm of finite element model}

To generate the finite element mesh with $3 \mathrm{D}$ meso-scale model, the following steps are applied by programming in FORTRAN:

1) Generate mesh elements of the specimen;

2) Calculate the central coordinates of each element;

3) Generate the randomly distributed aggregates using the method in Section 3.1; and

4) Check the position of each element with respect to that of each aggregate. If the element centre locates in one of the aggregates, assign the element with aggregate material; otherwise fill it with mortar material.

\subsection{Erosion technique}

Erosion is a technique used in FE modelling to overcome possible mesh tangling that causes simulation overflow when large deformation occurs. It is also used to simulate material failure. In LS-DYNA, there are several erosion algorisms available to remove elements under excessive distortions. In this study, after a parametric trial-and-error study, an erosion criterion depending on the maximum principal strain of 0.1 is used. Mortar and aggregate elements will be removed, when the principal strain of the element reaches the erosion criterion in the simulation. 
1

2 Spall tests were performed and reported in Wu et al (2005) in which Ø74-500 mm

3 concrete specimens with coarse aggregates were tested. Although the size of

4 aggregates were not mentioned by the authors, according to the representative

5 volumetric element (RVE) size, that is, the specimen size should be around 3 to 4

6 times of the size of each constituent in a composite material, it is reasonable to

7 assume the maximum aggregate size to be $16 \mathrm{~mm}$. The size of coarse aggregates

8 considered in the meso-scale model ranges from 4 to $16 \mathrm{~mm}$. This spall test is

9 numerically simulated to check the accuracy of the developed numerical model in 10 this study.

$11 \quad 4.1$ Numerical model

12 In the meso-scale model, concrete specimen is assumed to be a two-phase 13 composite material consisting of coarse aggregates with volume percentage $35 \%$ 14 and mortar matrix. Three series of coarse aggregates, namely $4-8 \mathrm{~mm}, 8-12 \mathrm{~mm}$ and $15 \quad 12-16 \mathrm{~mm}$, are considered in the numerical model. The dimension of the specimen is 16 the same as that in Wu et al (2005), that is, diameter $74 \mathrm{~mm}$ and length $500 \mathrm{~mm}$. To 17 save the computational effort, the striker bar and incident bar are not included in 18 numerical simulation, but only the concrete specimen itself is considered with the 19 stress boundary given in Wu et al (2005) being applied on the incident end of the 20 specimen as input. The 3D meso-scale model is shown in Fig. 4 while the stress 21 boundary obtained from Wu et al (2005) is shown in Fig. 5.

(a) 3D meso-scale model

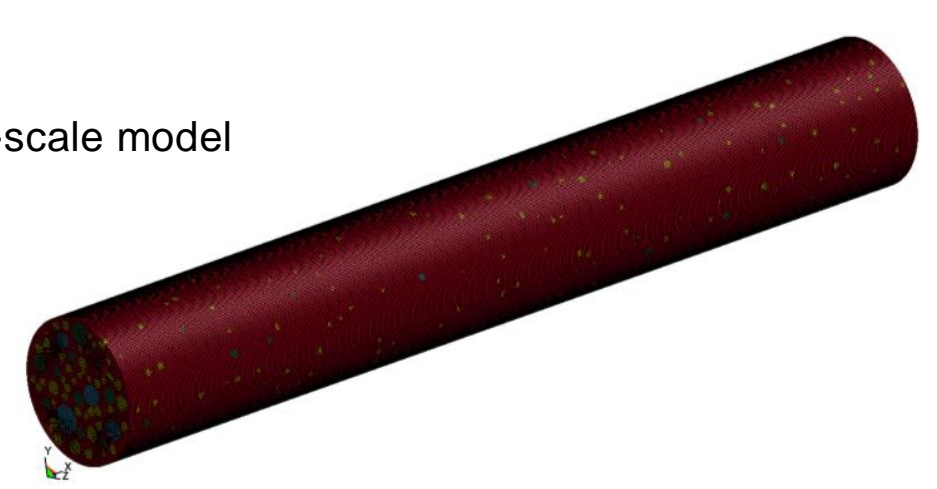

(b) mortar element

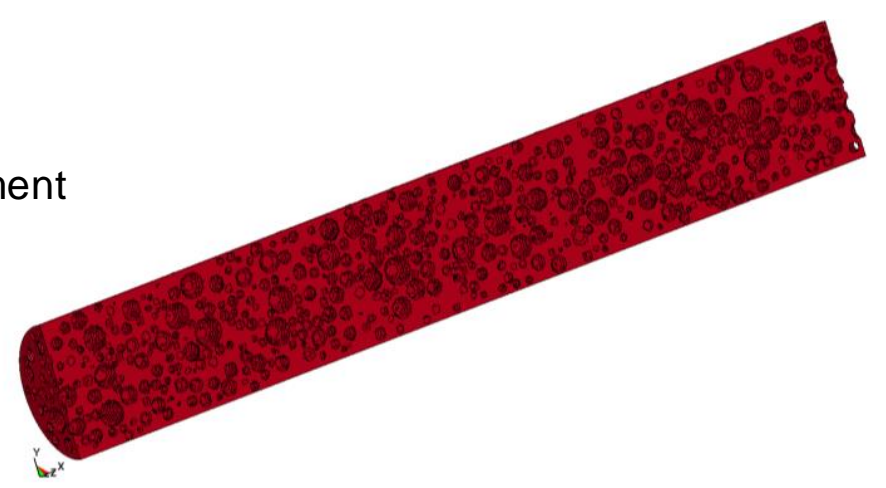

9 


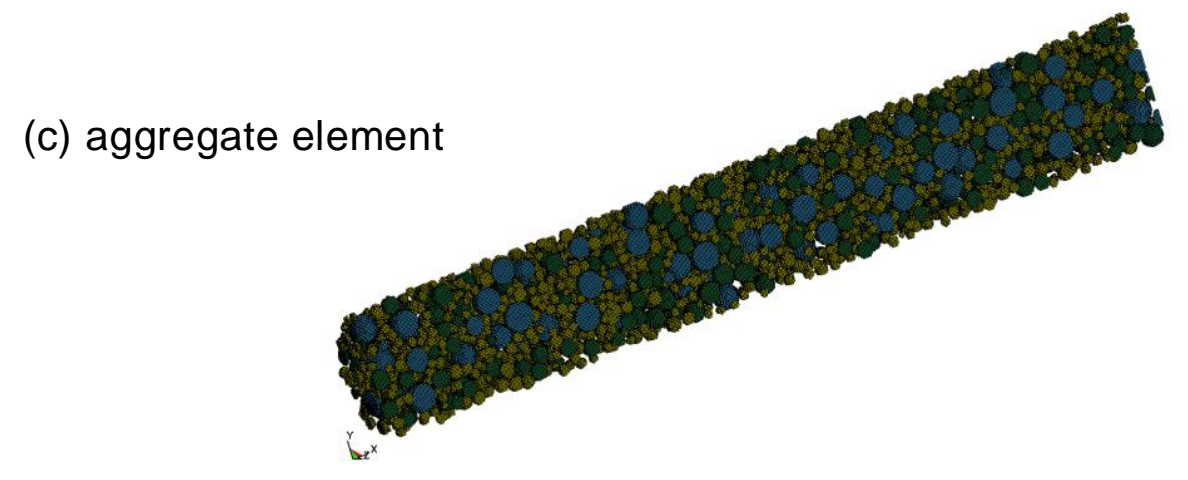

Figure 4: Finite element grid of 3D mesoscale model of spall test

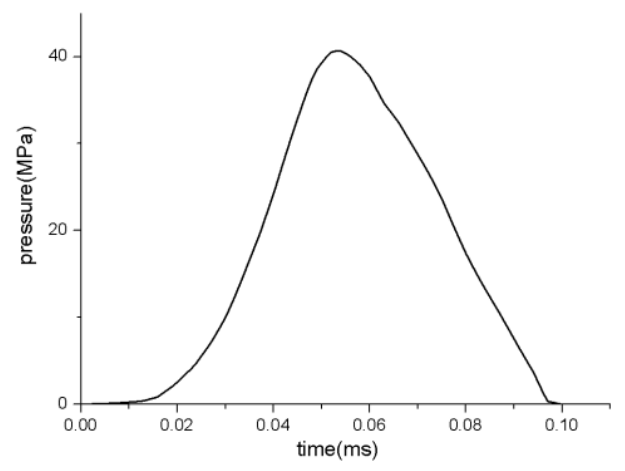

Figure 5: Compressive impulse as stress boundary

\subsection{Mesh convergence tests}

5

In the finite element analysis, the mesh size controls the computational time and the calculation accuracy. To optimize the effects of these two factors, a mesh sensitivity test is carried out for the meso-scale numerical model. Four mesh sizes, namely, $4 \mathrm{~mm}, 2 \mathrm{~mm}, 1 \mathrm{~mm}$ and $0.5 \mathrm{~mm}$, are used. The simulation convergence is examined by comparing the impact force at a cross section 150 $\mathrm{mm}$ from the incident end of the specimen as shown in Fig. 6. From the figure, it can be seen that $1 \mathrm{~mm}$ mesh size gives almost the same prediction compared to the simulation using mesh size $0.5 \mathrm{~mm}$ whereas simulations considering larger element sizes give slightly different predictions.

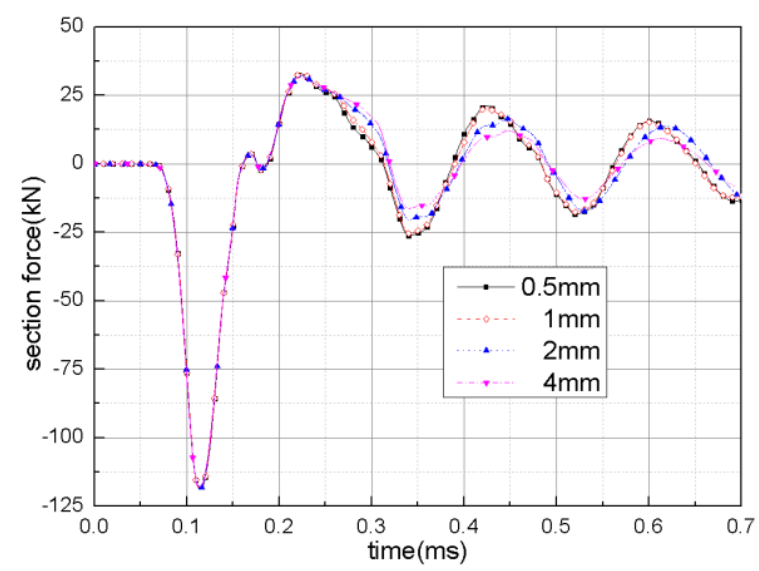

Figure 6: Force at cross section $150 \mathrm{~mm}$ from the incident end 


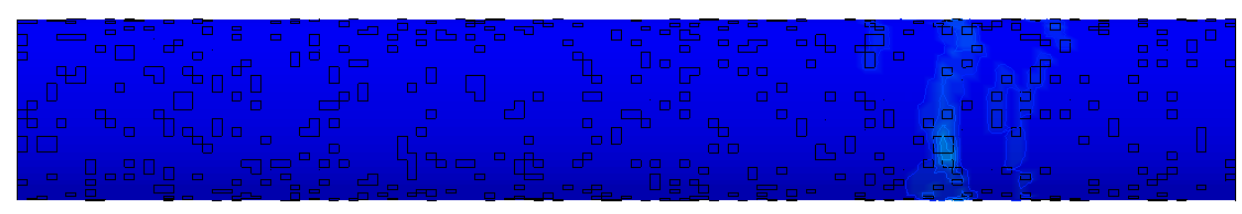

a) $4 \mathrm{~mm}$
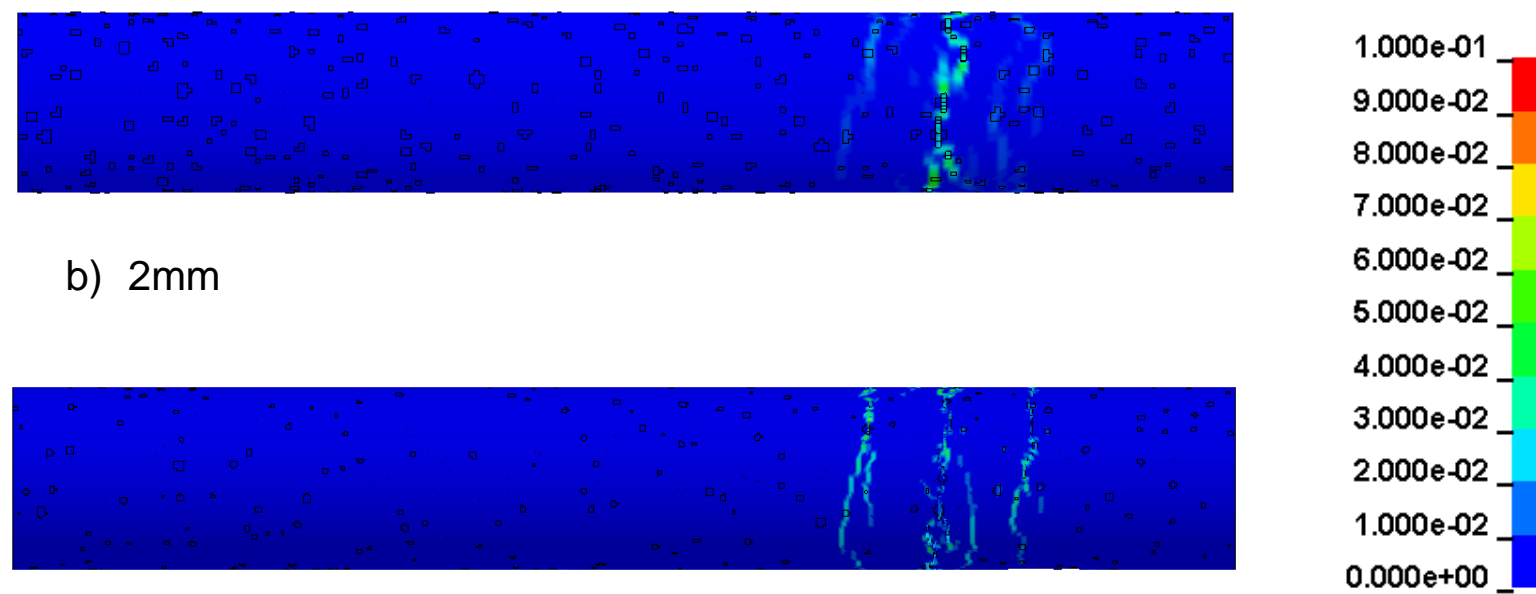

c) $1 \mathrm{~mm}$

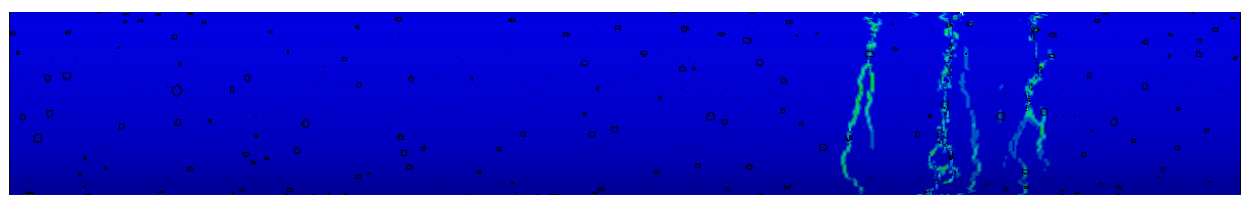

d) $0.5 \mathrm{~mm}$

Figure 7: Tensile strain of the specimen

The final tensile strains of the specimen obtained with the four mesh sizes are shown in Fig. 7. Because the fracture of concrete material in spall test is very localized, i.e., crack initiation will cause quick tensile strain relief in the adjacent elements, using relatively coarser mesh sizes, i.e. $4 \mathrm{~mm}$ and $2 \mathrm{~mm}$, cannot reliably predict the location of the fractures as shown in Figs. 7a and 7b. With $1 \mathrm{~mm}$ mesh size, the calculated strain concentrations tend to be the same as using $0.5 \mathrm{~mm}$ mesh size (Figs. 7c and 7d). The comparison of computational time of the four meshes is shown in Fig. 8. The cost of computation increases exponentially with the decrease of mesh size. Considering the accuracy and the efficiency in simulation, the finite element model with mesh size of $1 \mathrm{~mm}$ is used in this study. 


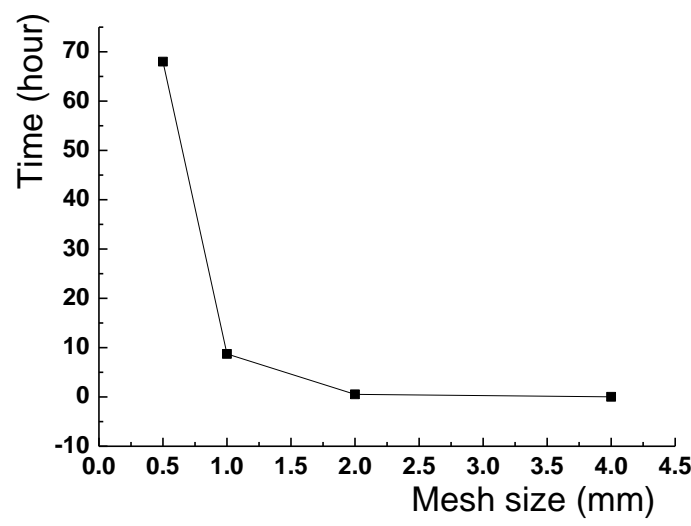

Figure 8: Comparison of computation time of four meshes

\subsection{Comparison of experimental and numerical results}

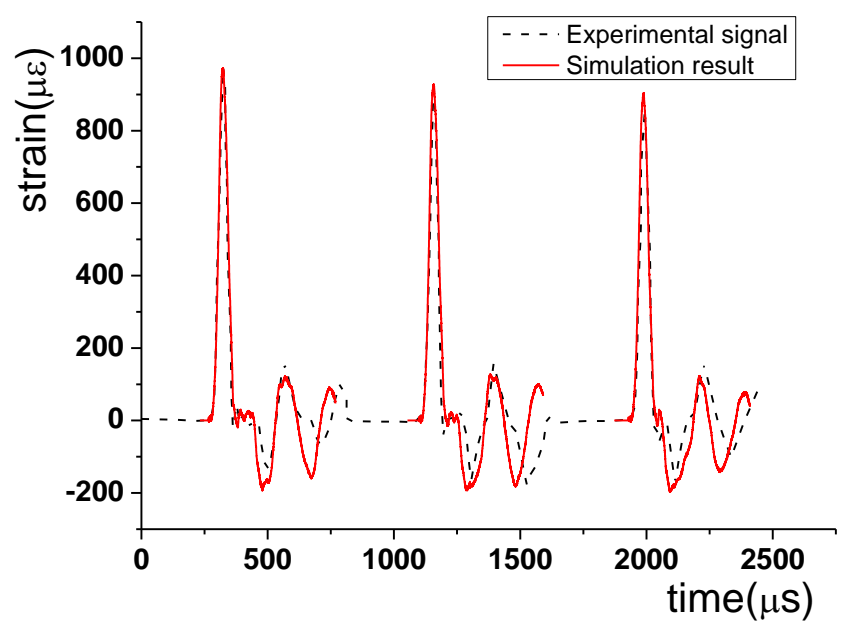

Figure 9: Comparison of strain histories at 150, 200 and $250 \mathrm{~mm}$ from the incident end

In the tests reported by Wu et al (2005), three strain gauges were attached on each specimen at 150, 200 and $250 \mathrm{~mm}$, respectively, from the incident end. The measured strain histories under the applied impulse in Fig. 5 are illustrated in Fig. 9. In the numerical simulation, strain histories of elements at the same locations as in the test are recorded for comparison, and the results are shown in Fig. 9. The corresponding failure patterns from the test and numerical simulation are compared in Fig. 10. As can be noticed the numerical simulation also successfully predicts the fracture location of the specimen. In spall tests, the tensile failure occurs owing to the superposition of the reflected tensile pulse and the incident compressive pulse. Therefore the fracture location depends on the incident compressive pulse shape. From the comparison of the strain histories in Fig. 9 and failure patterns in Fig. 10, it can be observed that under the same loading condition, the numerical simulation results yield good agreement with the test results, indicating the reliability of the material model adopted in the present study. 
(a)

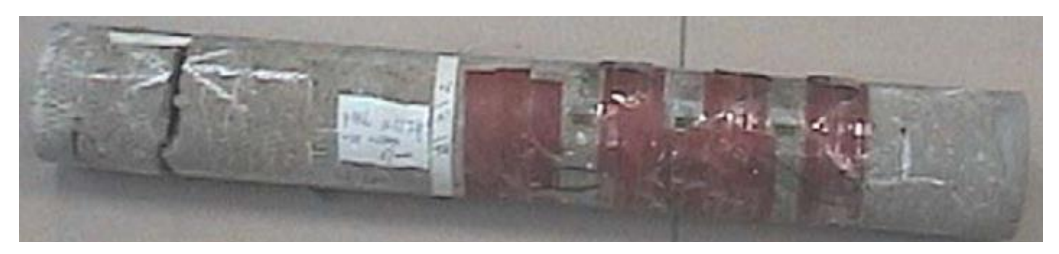

(b)

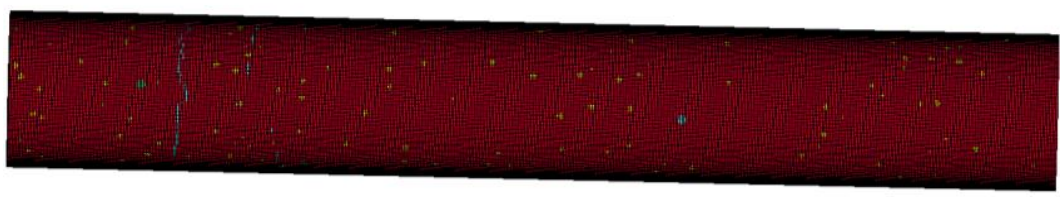

Figure 10: Comparison of failure patterns obtained from (a) experiment and (b) simulation

\section{ANALYSIS AND DISCUSSIONS}

\subsection{Influence of coarse aggregates on the measured strain signal}

In the spall test with relatively low velocity impact in Wu et al (2005), it was found that the incident compressive wave attenuates with its propagation direction. However, it is interesting to note that the wave attenuation in the reflected tensile impulse did not occur. Instead, the amplitude of the reflected tensile wave increased with the travelling distance as shown in Fig. 11a. With the similar loading condition adopted in the meso-scale concrete model, this phenomenon can also be found in the numerical simulation results as shown in Fig. 11b. This phenomenon was explained by the authors as the effects of damage growth that suppressed the attenuation effects. However, as shown in Fig. 12, the scaled damages, defined as $2 \lambda /\left(\lambda+\lambda_{m}\right)$ where $\lambda$ is the effective plastic strain which occurs at Point 1 and $\lambda_{m}$ is the effective plastic strain at the maximum strength at Point 2 in Fig. 2, calculated in numerical simulation inside the specimen only occurred in small scattered areas, and the level of damage is relatively small. Therefore the explanation by Wu et al (2005) that damage growth caused the reflected tensile stress wave increase with the propagation distance might not be exactly correct.

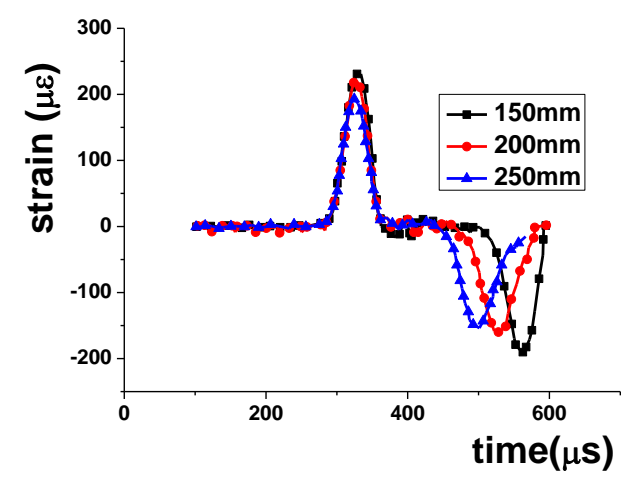

(a)

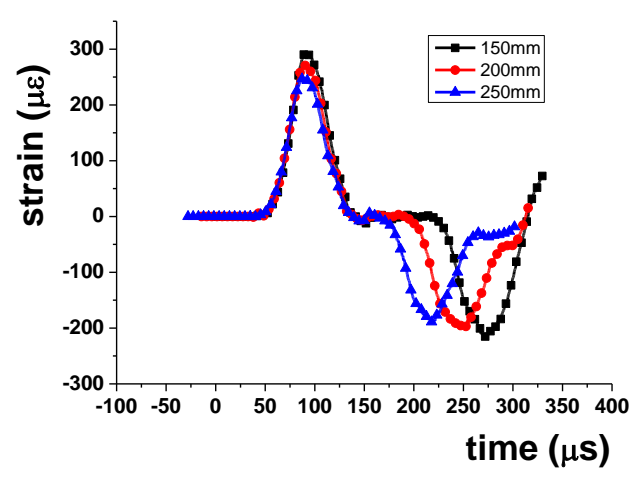

(b)

21 Figure 11: Comparison of strain histories from (a) experiment and (b) simulation 


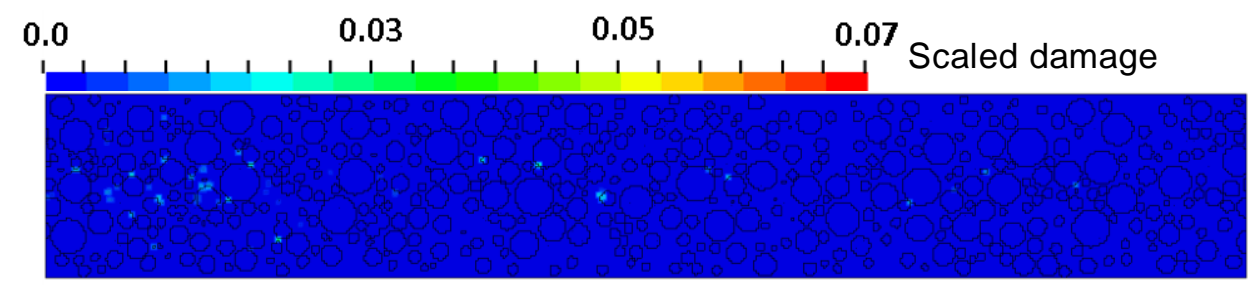

Figure 12: Scaled damage inside the specimen

It should be noted that coarse aggregates were used to prepare the concrete specimen in tests carried out by $\mathrm{Wu}$ et al. (2005). It is reasonable that the existence of coarse aggregates significantly increases the level of heterogeneity, which not only influences the stress wave propagation but also might affect the measured data, and thus caused the phenomenon observed in Fig. 11. To confirm this, strain histories of several surface elements at the cross section 200 $\mathrm{mm}$ from the loading surface (Fig. 13a) are obtained and compared in Fig. 13b. It can be seen that at the same cross section, the maximum strain in mortar increases with the distance to the aggregate element because of the lower elastic modulus of mortar than aggregates. Since the strain gauge used in the test only measures the averaged strain under a limited area on the specimen, there is a high possibility that the strain gauges at cross sections B and C (200 and $250 \mathrm{~mm}$ from the incident surface of the specimen, Fig. 11a) were attached on coarse aggregates whereas the gauge at cross section $A(150 \mathrm{~mm}$ from incident end) is on mortar. Because of the relatively low modulus, the measured strain on mortar is higher, which is the case for the numerical results shown in Fig.11b. To further demonstrate this assumption, the same numerical simulation using mortar material only is performed, and the simulation results are shown in Fig. 14. As shown, when there is no aggregate, the strain histories of the surface elements at the same cross section are almost identical (Fig. 14a); and the reflected tensile stress wave attenuation with the propagation distance can be found in Fig. 14b.

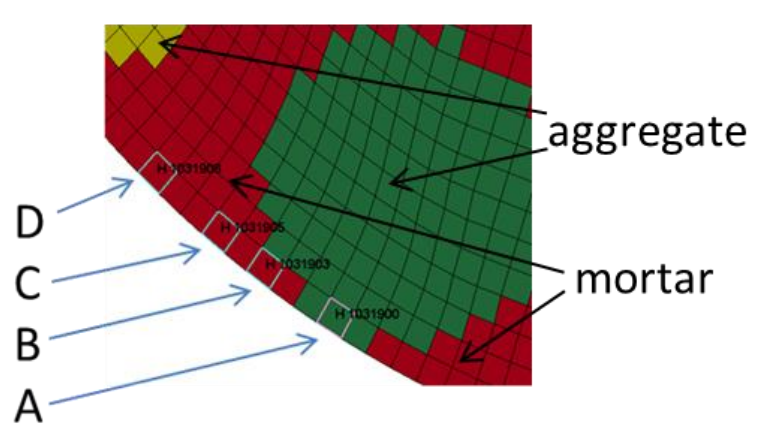

(a) Positions of elements

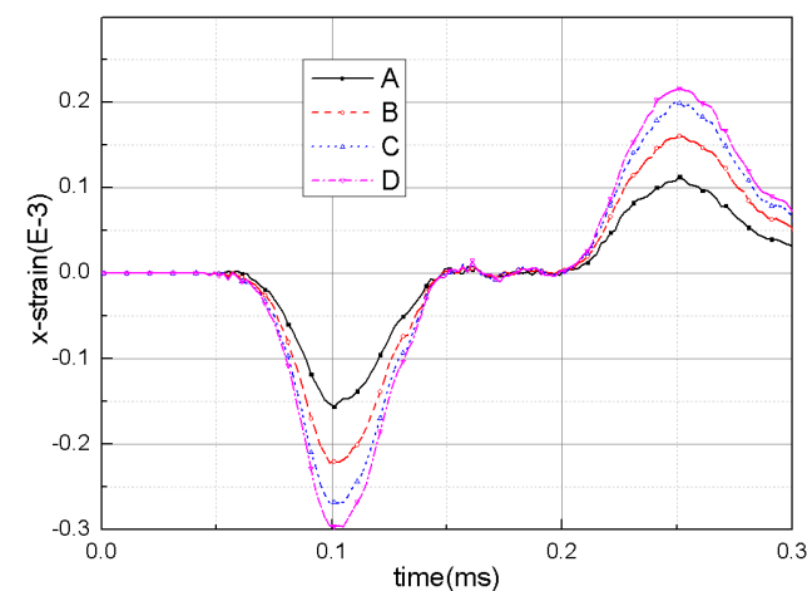

(b) Strain histories

Figure 13: Comparison of strain histories at a cross section 


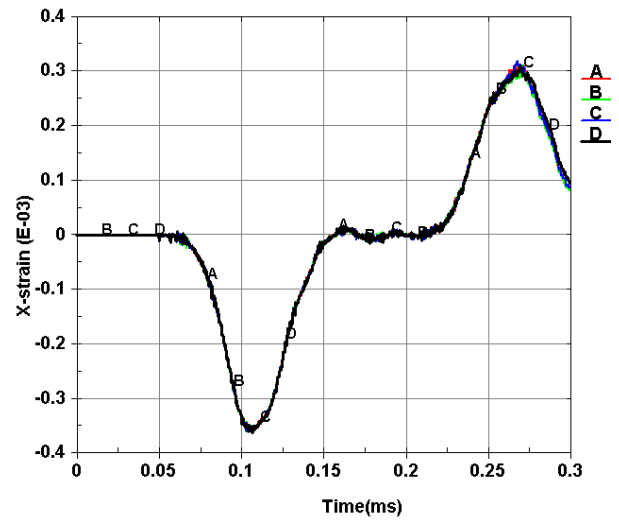

(a) Strain histories at a cross section

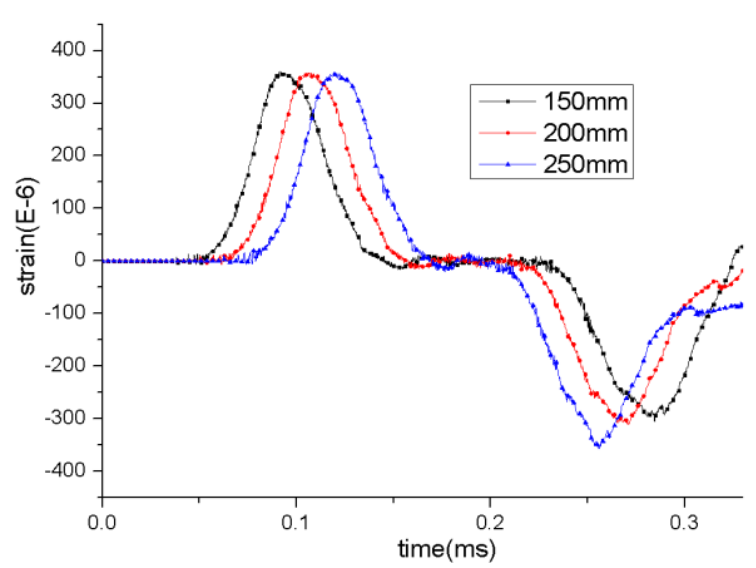

(b) strain histories at different sections with homogeneous mortar material

The above results indicate that when testing concrete material in spall tests, the existence of coarse aggregates has significant influence on the locally measured signal, and it is suggested to use more number of strain gauges at one cross section to derive the averaged strain signal so that the global behaviour of the concrete specimen can be more properly analysed.

\subsection{Wave attenuation analysis in spall tests}

Since the dynamic strength of concrete specimen in spall test is obtained by wave propagation, reflection and superposition, the wave dispersion and attenuation effects on wave propagation must be taken into consideration. Most previous studies focused on the geometric dispersion in spall tests of concrete materials (Klepaczko and Brara 2001; Brara et al. 2001,2006; Wu et al. 2005). Besides the geometric dispersion, concrete exhibits frequency dependent attenuation behaviour because of the heterogeneity due to the existence of coarse aggregates (Eric and Surendra, 1995; Philippidis and Aggelis, 2005). To give quick assessments of the wave attenuation level that can be used in laboratory test data analysis, a series of compressive stress waves with the same amplitude but different wavelengths, equivalent to different frequencies, are applied to the specimen as shown in Fig. 15. The peak stress is set to be $8 \mathrm{MPa}$ so that it does not result in any compressive damage to the concrete specimen during the compressive stress wave propagation.

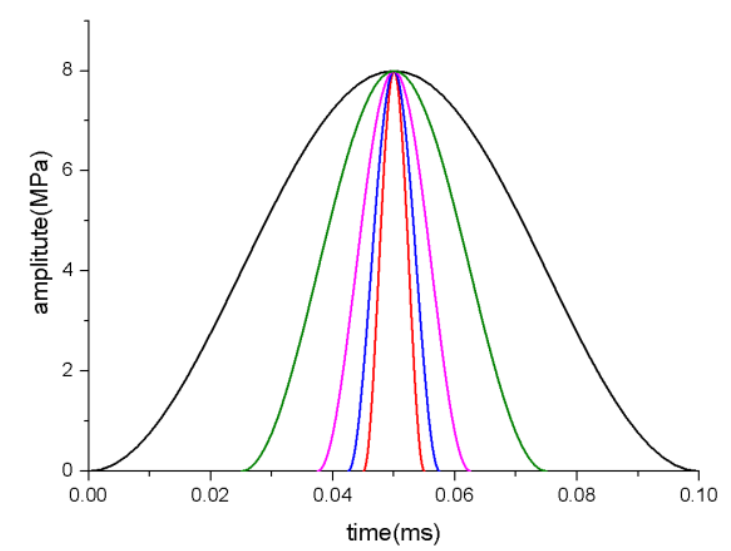

Figure 15: Input compressive pulse curves 
1 Using the input waves with the same amplitude but different wavelengths 2 shown in Fig. 15, the simulation results are shown in Fig. 16a where $\bar{\lambda}$ is a 3 dimensionless wavelength which is the ratio of wavelength to specimen diameter. 4 It can be observed that when $\bar{\lambda}$ is greater than 3 , the wave attenuation is 5 negligible. The change of stress amplitude with respect to spatial distance can be described by the equation (Wang 2007)

$$
\sigma=\sigma_{0} e^{-\alpha x}
$$

where $\sigma_{0}$ is the peak input stress, $x$ is the distance to the incident end of the concrete specimen, and $\alpha$ is the attenuation exponent which is dependent on $\bar{\lambda}$.

By fitting the simulation results using Equation (18) shown in Fig. 16a, different attenuation exponent can be derived. The derived values of $\alpha$ with respect to the dimensionless parameter $\bar{\lambda}$ are plotted in Fig. $16 \mathrm{~b}$, and the fitted curve can be expressed as

$$
\alpha=0.00204 e^{-0.331 \bar{\lambda}^{2}}
$$

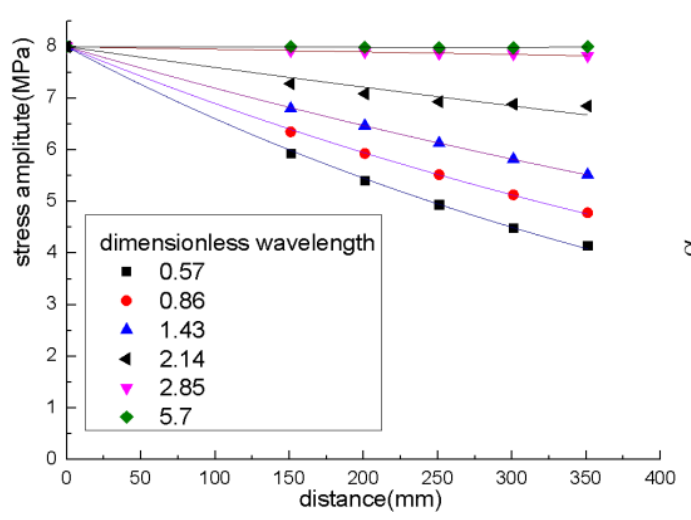

(a) attenuation of different wavelength pulses

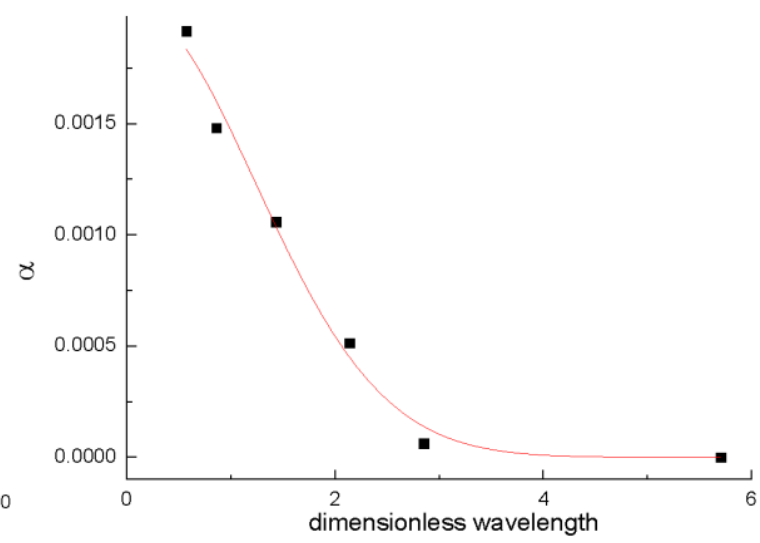

(b) attenuation exponent varies with wavelength

Figure 16: Wave attenuation with dimensionless wavelength

Equations (18-19) can be used to give quick analysis of the spall test data, i.e., if the input wavelength and specimen diameter are known, $\bar{\lambda}$ can be obtained and substituted into Equation (19) to derive the attenuation exponent $\alpha$, which can be substituted into Equation (18) to calculate the wave attenuation.

\subsection{Quantification of contributions of aggregates to strength in spall tests}

Using the above numerical model, spall tests of concrete specimens with $35 \%$ volume of coarse aggregates are simulated. As has been mentioned, most of the experimental studies used mortar material to prepare the specimen, and the test results are used to describe concrete material properties. This may result in inaccurate concrete dynamic material properties in tension at high strain rates. To confirm this, numerical simulations using mortar material alone are also carried out, and the results are compared with those obtained from simulations considering meso-scale model. 


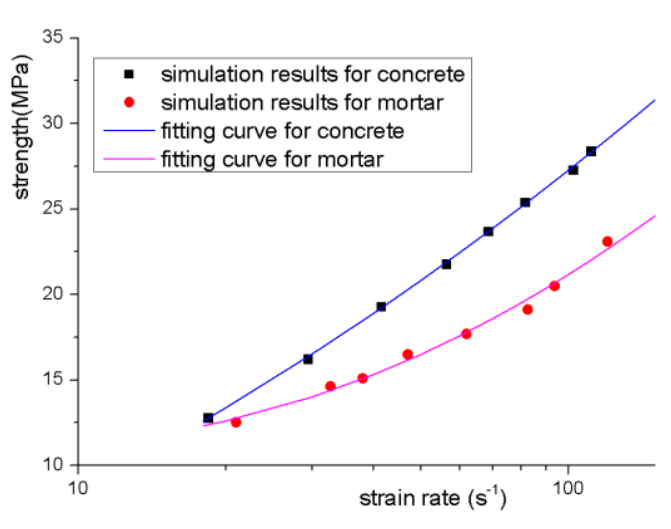

Figure 17: Comparison of dynamic strengths

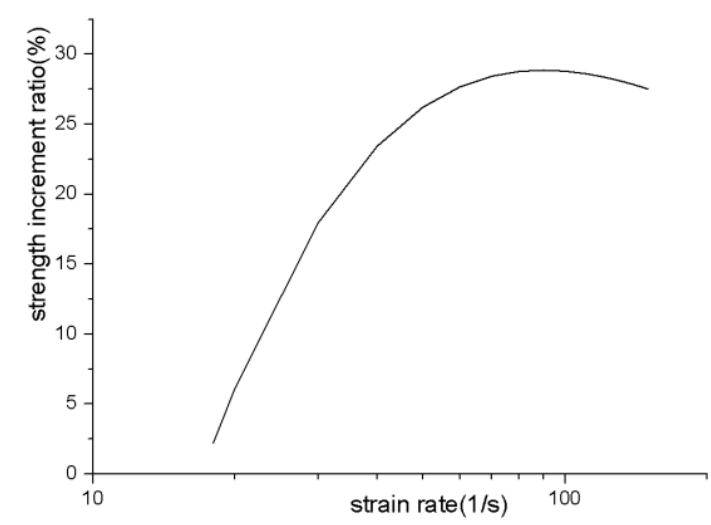

Figure 18: Strength increment ratio

Fig. 17 shows the dynamic tensile strengths of concrete and mortar specimens obtained from the simulations under varying strain rates from $201 / \mathrm{s}$ to $150 \mathrm{1} / \mathrm{s}$. As shown, all strengths obtained from concrete specimen with $35 \%$ aggregates are apparently higher than those from simulations of mortar specimen. This observation indicates the importance and necessity of including aggregates in the numerical and experimental studies of spall tests.

Since neglecting aggregates leads to underestimation of concrete dynamic tensile strength, e.g., based on Fig. 17, the difference of strengths obtained from concrete and mortar specimens is $6 \%$ and $25 \%$ under strain rate $201 / \mathrm{s}$ and 120 $1 / \mathrm{s}$, respectively, it is necessary to quantify the ratios of concrete strength to mortar strength. The numerically simulated concrete to mortar strength ratios respect to strain rates are plotted in Fig. 18. As shown, the strength ratio firstly increases rapidly with the strain rate. However, the increment becomes slow and steady at the strain rate about $501 / \mathrm{s}$, and even drops slightly at the strain rate of $90 \mathrm{1} / \mathrm{s}$. This is because, as mentioned in Section 2.4, the tensile DIF of aggregates is set to have a constant value when the strain rate exceeds $501 / \mathrm{s}$ because of the very limited test data in the literature. When the strain rate is higher than $50 \mathrm{1} / \mathrm{s}$, the contribution of aggregates to strength increment remains unchanged with the strain rate whereas the strength of mortar material still increases with the strain rate. The fitted curve in Fig. 18 can be expressed by the equation

$$
\zeta=-0.543(\log \dot{\varepsilon})^{2}+2.123 \log \dot{\varepsilon}-1.78 \quad \text { for } \quad 20 s^{-1}<\dot{\varepsilon}<150 s^{-1}
$$

In practice, Equation (20) can be used to modify the strength of mortar materials obtained in spall tests to derive the strength of concrete material with $35 \%$ coarse aggregates.

\section{CONCLUSIONS}

This study uses meso-scale model to numerically investigate the behaviour of concrete material at high strain rates in spall tests. Because coarse aggregates are stronger than the mortar matrix with higher elastic modulus, they have considerable influence on the locally (strain gauge) measured signals, which 
might give inaccurate or even misleading test results. Thus it is suggested to use more number of strain gauges at one cross section to derive the averaged strain signal for proper analysis of the global behaviour of the concrete specimen in spall tests. While previous studies only considered the geometric wave attenuation in analysing the test results, this study also analysed the wave frequency-dependent attenuation in spall tests, and proposed analytical formulae that enable quick analyses of the experimental data. It is found that including aggregates in the concrete specimen results in a higher dynamic strength. An empirical relation is proposed to modify the results obtained from spall tests of mortar specimens without aggregates.

\section{ACKNOWLEDGEMENTS}

The first author would like to acknowledge China National Natural Science Foundation (Grant no.: 51174173 and 11102195) for financial support to carry out this study. The second and third authors would like to acknowledge Australian Research Council (Grant no.: DP130104332) for financial support to carry out this study.

\section{REFERENCES}

Brara A, Camborde F, Klepaczko JR and Mariotti C (2001). Experimental and numerical study of concrete at high strain rates in tension. Mech Mater 33: 33-45.

Brara A, Klepaczko JR (2006). Experimental characterization of concrete in dynamic tension. Mech Mater 38: 253-267.

Daimaruya M, Kobayashi H, Nonaka T (1997). Impact tensile strength and fracture of concrete. Proceedings of the International Conference EURODYMAT 97. J Phys Coll C3:C3-253.

Landis EN, Shah SP (1995). Frequency-dependent stress wave attenuation in cement-based materials. J Eng Mech 121(6): 737-743.

Erzar B, Forquin P (2010). An Experimental Method to Determine the Tensile Strength of Concrete at High Rates of Strain. Exp Mech 50: 941-955.

Gálvez Díaz-Rubio F, Rodríguez Pérez J, Sánchez Gálvez V (2002). The spalling of long bars as a reliable method of measuring the dynamic tensile strength of ceramics. Int $J$ Impact Eng 27: 161177.

Gomez JT, Shukla A and Sharma A (2001). Static and dynamic behavior of concrete and granite in tension with damage. Theor Appl Fract Mech 36: 37-49.

Hao $\mathrm{Y}, \mathrm{Hao} \mathrm{H}$ (2011). Numerical evaluation of the influence of aggregates on concrete compressive strength at high strain rate. Int J Prot Struct 2(2): 177-206.

Hao Y, Hao H (2013), Numerical investigation of the dynamic compressive behaviour of rock materials at high strain rate. Rock Mech Rock Eng, 46(2): 373-388.

Hao Y, Hao H, Jiang GP, Zhou Y (2013). Experimental confirmation of some factors influencing dynamic concrete compressive strengths in high-speed impact tests, Cem Con Res 52: 63-70.

Hao Y, Hao H, Li ZX (2009). Numerical Analysis of Lateral Inertial Confinement Effects on Impact Test of Concrete Compressive Material Properties. Int J Prot Struct 1(1): 145-167.

Hao Y, Hao H and Zhang X (2012). Numerical analysis of concrete material properties at high strain rate under direct tension. Int $\mathrm{J}$ Impact Eng 39: 51-62.

Jones J, Wu C, Oehlers DJ, Whittaker AS, Sun W, Marks S and Coppola R (2009). Finite difference analysis of simply supported RC slabs for blast loading. Eng Struct 31: 2825-2832.

Klepaczko JR, Brara A (2001). An experimental method for dynamic tensile testing of concrete by spalling. Int J Impact Eng 25: 387-409.

LSTC (2007). LS-DYNA version 971 keyword user's manual. Livermore Software Technology Corporation, California, USA.

Malvar LJ, Crawford JE, Wesevich JW (1997). A plasticity concrete material model for Dyna3D. Int J Impact Eng 19: 847-873.

Malva LJ, John E. Crawford(1998). DYNAMIC INCREASE FACTORS FOR CONCRETE, TwentyEighth DDESB Seminar, Orlando, FL. 
1 Philippidis TP, Aggelis DG (2005). Experimental study of wave dispersion and attenuation in concrete. Ultrasonics 43: 584-595.

Schuler H, Mayrhofer C and Thoma K (2006). Spall experiments for the measurement of the tensile strength and fracture energy of concrete at high strain rates. Int J Impact Eng 32: 1635-1650.

Tedesco JW, Ross CA, McGill PB and O'Neil BP (1991). Numerical analysis of high strain rate concrete direct tension tests. Comput Struct 40(2), pp. 313-327.

Wang LL. Foundations of Stress Waves (National Defense Industry Press, China, 1985; 2nd Edition, 2005; English Edition, Elsevier, Amsterdam, 2007).

Weerheijm J, Van Doormaal JCAM (2007) Tensile failure of concrete at high loading rates: new test data on strength and fracture energy from instrumented spalling tests. Int J Impact Eng 34: 609626.

Wu HJ, Zhang QM, Huang FL, Jin QK (2005). Experimental and numerical investigation on the dynamic tensile strength of concrete. Int J Impact Eng 32: 605-617.

Yan DM and Lin G (2006). Dynamic properties of concrete in dynamic tension. Cem Con Res 36: 1371-1378.

Zhang L, Hu SS, Chen DX, Yu ZQ, Liu F(2009). An Experimental Technique for Spalling of Concrete, Exp Mech 49: 523-532.

Zhou X, Hao H (2008a). Mesoscale modeling of concrete tensile failure mechanism at high strain rate. Int J Comput Struct 86: 2013-2026.

Zhou X, Hao H (2008b). Modelling of compressive behaviour of concrete-like materials at high strain rate. Int J Solids Struct 45(17): 4648-4661. 